\title{
Neumonitis granulomatosa por instilación endovesical de BCG
}

\author{
Gómez-Ferrer Lozano Á, Navarro Antón JA, Sala Aznar A, Mola Arizo MaJ, Gonzalvo Pérez V, \\ Cantó Faubel E, Polo i Peris AC.
}

Servicio de Urología, Hospital Lluís Alcanyís de Xàtiva, Valencia.

Actas Uro Esp. 2006;30(8):839-842

\section{RESUMEN}

NEUMONITIS GRANULOMATOSA POR INSTILACIÓN ENDOVESICAL DE BCG

Presentamos dos casos de afectación pulmonar por BCG diagnosticados recientemente en nuestro servicio. Un primer paciente varón de 81 años de edad que desarrolló un cuadro de neumonitis intersticial granulomatosa severa en el contexto de un tratamiento mediante instilación endovesical de BCG por tumor vesical de alto grado. Este paciente fue tratado mediante corticoterapia y triple asociación de antituberculosos con una respuesta rápida y completa. El segundo caso fue un hallazgo radiológico en un paciente de 56 años que presentaba fiebre persistente como único síntoma y que mejoró tras iniciar doble asociación de tuberculostáticos.

Palabras clave: BCG. Neumonitis intersticial. Complicaciones.

\section{ABSTRACT \\ GRANULOMATOUS PNEUMONITIS SECONDARY TO BCG BLADDER INSTILLATION}

We report two cases of pulmonary BCG-induced pneumonitis recently diagnosed amongst our patients. The first case is a 81 year old man under BCG bladder instillation treatment because of high grade superficial bladder cancer who developed a severe interstitial granulomatous pneumonitis. The patient was treated with corticosteroids and tuberculostatics with a rapid and complete response. The second patient is a young man who presented only with persistent fever and also had a complete response after treatment.

Keywords: BCG. Interstitial pneumonitis. Complications.

$\mathrm{E}^{1}$ tratamiento mediante instilaciones endovesicales de BCG es una modalidad terapéutica con eficacia demostrada en tumores vesicales de alto grado y carcinoma in situ. Se utiliza con mucha frecuencia en los servicios de urología de todo el mundo, con una variabilidad de esquemas y calendarios posológicos. Sin embargo, su empleo no está exento de complicaciones, siendo las más frecuentes las de aparición local, como la cistitis granulomatosa. En menor medida se manifiestan sintomas y signos sistémicos y muy raramente afectación de otros órganos como riñón, hígado o pulmón ${ }^{1}$.

\section{CASOS CLÍNICOS}

Caso 1

Comunicamos el caso de un paciente de 81 años de edad con antecedentes de fibrilación auricular que inició tratamiento con BCG endovesical por tumor vesical superficial de alto grado con la cepa RIVM.

Siete días después de la tercera instilación manifiesta de forma insidiosa mal estado general, febrícula, astenia, anorexia y disnea a leves esfuerzos sin tos ni expectoración. Es valorado en urgencias practicándose exploraciones complementarias que pusieron de manifiesto la existen- 
cia de una fibrilación auricular a 110 latidos por minuto, saturación de oxígeno ambiental a 91\% y patrón difuso intersticial bilateral en la $\mathrm{Rx}$ de tórax (Fig. 1) con crepitantes bibasales a la auscultación. El paciente fue tratado ambulatoriamente con digitálicos y diuréticos sin respuesta. Tras consultar con el servicio de Urología dados sus antecedentes se cursaron cultivos para micobacterias en sangre, orina y esputo -que resultaron todos ellos negativos- , se suspendió el tratamiento con BCG y se remitió al servicio de Neumología, solicitando TAC torácico y broncoscopia. En el TAC se observó infiltración intersticial difusa (Fig. 2). Durante la broncoscopia se realizó lavado broncoalveolar para identificación

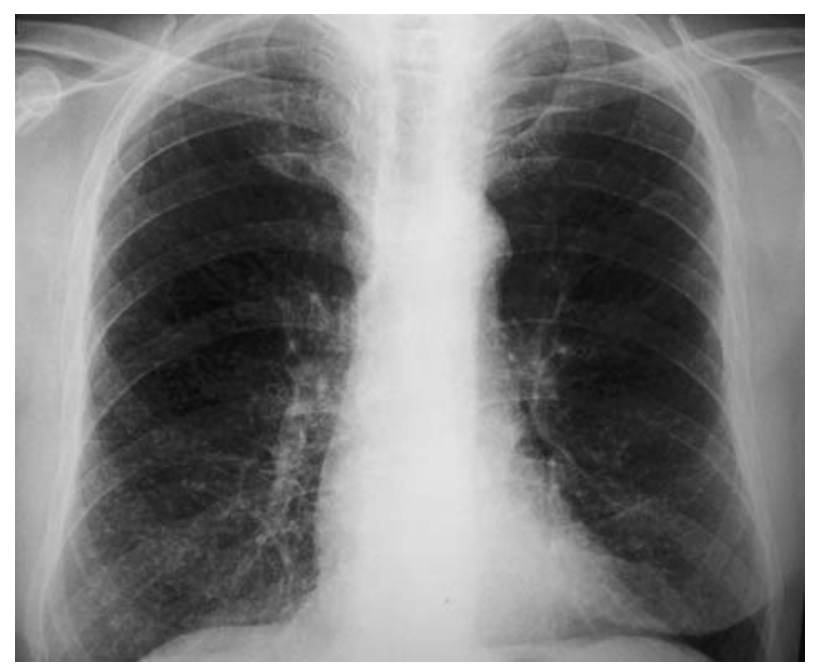

FIGURA 1. Radiografía de tórax: Patrón intersticial micronodular difuso. de micobacterias que fue negativa y citología con hallazgo de células de respuesta inflamatoria aguda. La biopsia transbronquial puso de manifiesto la existencia de granulomas similares a los existentes en la TBC pulmonar clásica (Fig. 3).

Ante la sospecha de neumonitis intersticial granulomatosa por BCG se instauró tratamiento con corticoides (prednisona $45 \mathrm{mg} /$ día) y triple asociación de rifampicina, isoniazida y pirazinamida durante dos meses y rifampicina + isoniazida dos meses más hasta completar cuatro meses de tratamiento con el mismo esquema que en una TBC primaria.

La respuesta fue espectacular en los primeros días, desapareciendo la fiebre y la disnea al quinto día de tratamiento y con normalización de la imagen radiográfica practicada a las cuatro semanas. El paciente se encuentra completamente asintomático a los 6 meses del diagnóstico.

\section{Caso 2}

Tres meses después un varón de 56 años sin otros antecedentes inició tratamiento con BCG por carcinoma in situ con la misma cepa y esquema posológico que el paciente 1 . Después de la cuarta instilación comienza a tener fiebre alta entre 38 y $39{ }^{\circ} \mathrm{C}$ de forma persistente y monosintomática, con escasa repercusión del estado general y sin manifestaciones pulmonares. Se interrumpe el esquema pautado de tratamiento pero la fiebre persiste por lo que a la semana se inicia tratamiento con isoniazida y antitérmicos. El paciente continúa con fiebre una semana después motivo por el que se añade rifampicina y se solicita radio-
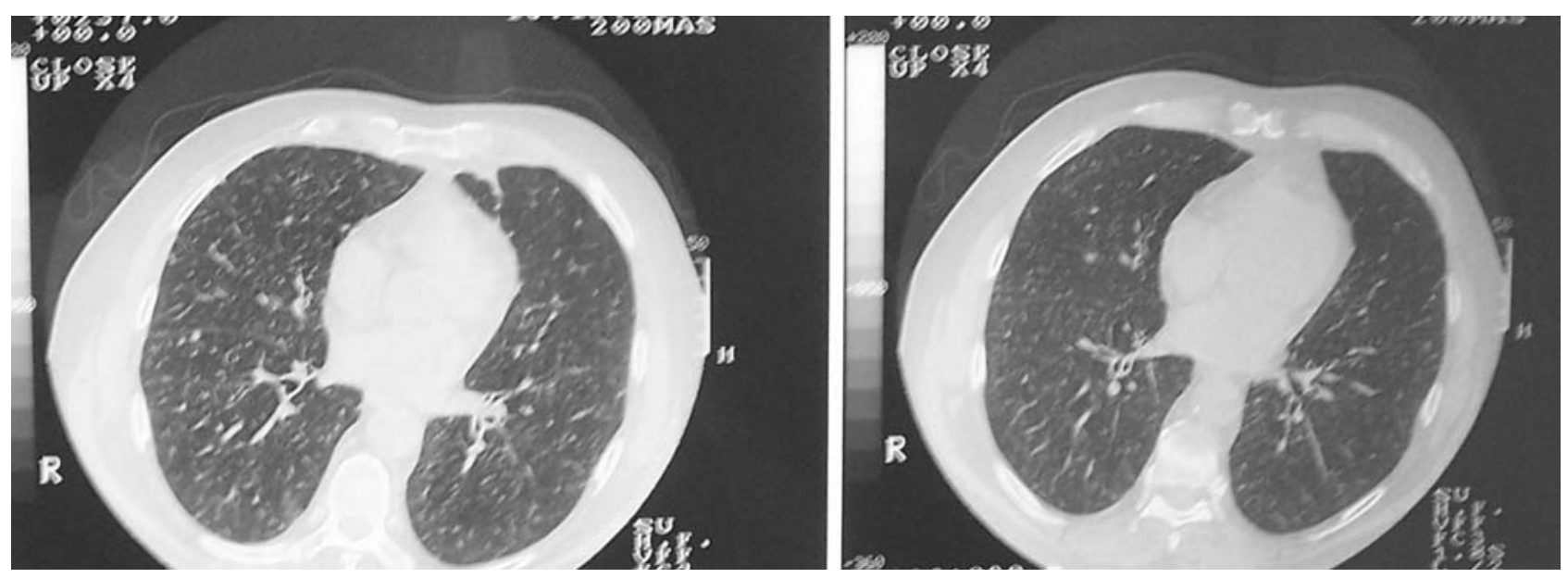

FIGURA 2. TAC torácico: Infiltrado micronodulillar. 


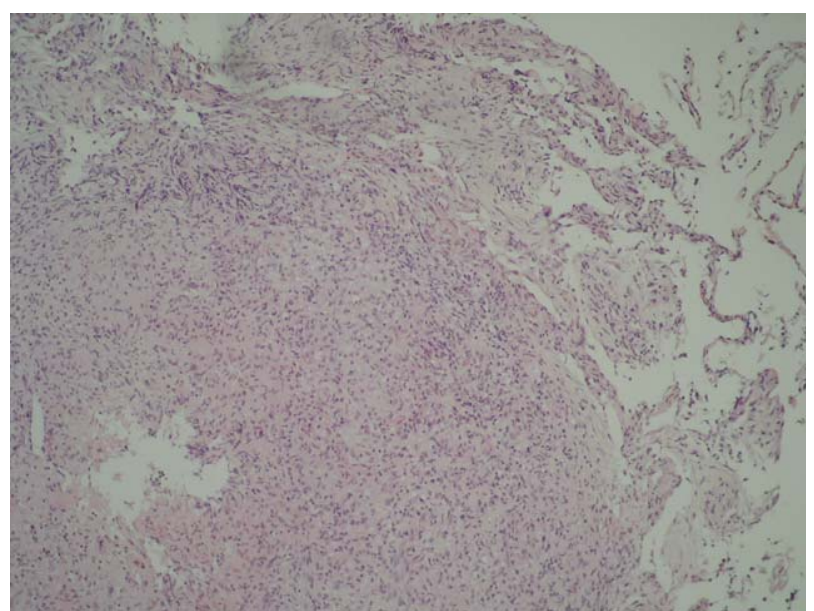

FIGURA 3. Formación de granuloma con abundantes histiocitos y células gigantes multinucleadas.

grafia de tórax de manera ambulatoria. La radiografia y la TAC torácica ponen de manifiesto una infiltración difusa bilateral con patrón micronodulillar sugestivo de neumonitis granulomatosa muy similar a la del paciente 1 . El paciente es remitido a neumología, que ante la buena respuesta al doble tratamiento, no considera necesaria la realización de más exploraciones complementarias ni la asociación de corticoides. El paciente completa cuatro meses de tratamiento y en la actualidad está completamente asintomático.

\section{DISCUSIÓN}

Los efectos secundarios del tratamiento endovesical con BCG son frecuentes, pero principalmente relacionados con la respuesta inflamatoria local en forma de síndrome miccional irritativo, disuria, hematuria y polaquiuria.

La aparición de complicaciones sistémicas en el transcurso de un tratamiento endovesical con BCG está ampliamente documentado y se estima que aparece hasta un $5 \%$ de los $\operatorname{casos}^{1,2}$. La manifestación más frecuente es la fiebre que aparece en un 3\% de los pacientes, y cursa de manera autolimitada en la mayoría de los casos. Sin embargo, la afectación parenquimatosa es mucho menos frecuente, siendo posible en varios órganos (hígado, riñón) pero preferentemente en pulmón. La afectación parenquimatosa granulomatosa en hígado y/o pulmón se ha descrito en el 0,7\% de los casos. En pulmón ocasiona un cuadro de neumonitis con patrón radiográfico intersticial, acompañándose de manifestaciones sisté- micas como fiebre y síndrome constitucional ${ }^{3}$. La identificación de micobacterias en sangre, esputo o lavado broncoalveolar es muy difícil y el diagnóstico de certeza se establece mediante biopsia con hallazgos de granulomas similares a los de la TBC pulmonar clásica. La terapia con corticoides y tuberculostáticos se ha demostrado efectiva y nosotros seguimos el esquema terapéutico comunicado por Martín Escudero ${ }^{4}$ obteniendo una respuesta rápida, segura y estable en el tiempo.

Algunos autores consideran que la etiopatogenia de este cuadro se basa principalmente en una alteración de la respuesta inmune y justifican el tratamiento con corticoides, incluso llegando a postular dicho tratamiento como única medida ${ }^{5}$. No obstante, otros autores consideran esta actitud extremadamente peligrosa, pues aunque en la mayoría de las ocasiones no es posible identificar el bacilo, existen casos descritos de sepsis mortal por BCG y no se puede descartar la diseminación sistémica del bacilo ${ }^{6}$. Sólo en escasas ocasiones se ha logrado identificar el bacilo mediante cultivo, tinción o técnicas de PCR. Además la inmunosupresión inducida por corticoides justificaría por sí misma la profilaxis con antituberculosos. De todas formas, la reacción inmunológica juega un papel fundamental, pues la respuesta a la corticoterapia es más rápida que la que cabría observar con el tratamiento antituberculoso que precisa de más días para ver iniciado su efecto. En nuestro segundo paciente no se instauró corticoterapia ante la nula sintomatología respiratoria y evolucionó igualmente bien, clínica y radiológicamente, aunque la fiebre tardó más días en desaparecer, otra semana después de la doble asociación.

En la mayoría de los casos se describe un antecedente de alteración de la barrera urotelial, por sondaje traumático en dos pacientes y resección reciente de implante tumoral en uretra en otro. El paso directo de BCG al torrente sanguíneo facilitaría obviamente la diseminación hematógena así como la precocidad en la presentación de los síntomas que padecieron dichos enfermos. Es evidente que se indica la demora de tratamiento con BCG en dichas situaciones hasta la completa cicatrización del urotelio dañado. Sin embargo, en ninguno de nuestros dos pacientes hemos tenido antecedente alguno de agresión al urotelio vesical o uretral. 


\section{CONCLUSIONES}

Debemos sospechar neumonitis intersticial en todo paciente bajo tratamiento con BCG que se presente con un cuadro constitucional y sintomas respiratorios. El primer paso será la interrupción de la instilación con BCG y la práctica de exploraciones complementarias: radiografia/TAC y broncoscopia con biopsia, pues la búsqueda de microbacterias será muy difícil y no debe condicionar el retraso del tratamiento de esta complicación. La terapia con corticoides y antituberculosos según la pauta clásica de tratamiento de la TBC primaria se ha demostrado segura y eficaz en los casos consultados así como en los nuestros.

\section{REFERENCIAS}

1. Lamm DL. Complications of Bacillus Calmette-Guerin inmunotherapy. Urologic Clinics of North America. 1992 Aug;19(3):565-572.
2. Sakamoto GD, Burden J, Fisher D. Systemic bacillus Calmette-Guerin infection after transurethral administration for superficial bladder carcinoma. J Urol. 1989 Oct;142(4):1073-1074.

3. Diner EK, Verghese M. Interstitial pneumonitis secondary to intravesical bacillus Calmette-Guerin for carcinoma in situ of the bladder. International Braz J Urol. 2004 SepOct;30(5):400-402.

4. Martín Escudero JC, Pérez Paredes G, Asensio Sánchez T, Herreros Fernández V. Neumonitis granulomatosa por BCG. An Med Interna. 2003;20(2):57.

5. Molina JM, Rabian C, D`Agay MF, Modai J. Hipersensitivity systemic reaction following intravesical bacillus CalmetteGuerin: successful treatment with steroids. J Urol. 1992 March;147:695-699.

6. LeMense GP, Strange C. Granulomatous pneumonitis following intravesical BCG. What therapy is needed? Chest. 1994 Nov; 106(5):1624-1626.

Dr. Á. Gómez-Ferrer Lozano

E-mail: gomez_alvloz@gva.es

(Trabajo recibido el 8 de septiembre 2005) 